\title{
VI
}

\section{THE ROUTINE TREATMENT OF SYPHILIS AND GONORRHEA (IV)}

By Professor F. PINKUS, Berlin

\section{A. THE TREATMENT OF SYPHILIS}

Diagnosis.-I require a stringent microscopic examination as the basis for the diagnosis of primary syphilitic cases. Serum is taken from the suspected sore, and examined under the ultra-microscope, using dark ground illumination. The serum is then tested for the BordetWassermann reaction by the original method, the complement being provided by the Wassermann laboratory. The flocculation test employed is that of the TrübungsMethod of Meinicke.

Treatment.-The preparation of arsenic most used in my clinic is neo-salvarsan, but I also employ a number of others, including sodium-salvarsan, spirozid and stovarsol. I administer bismuth in the form of sorbismal and casbis, and iodine as the iodide of potassium. I have ceased to rely on mercurial preparations of any kind.

In treating an adult male patient of average weight whose serum reaction is negative I administer neosalvarsan according to the following time-table.

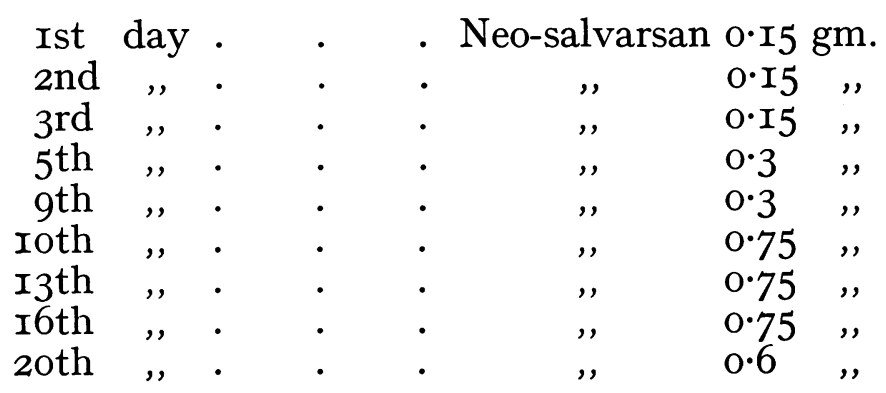

Thereafter two injections are given weekly, later increasing to three if tolerance is good, of anything up to 


\section{TREATMENT OF SYPHILIS AND GONORRHCEA}

0.9 gm. neo-salvarsan. The maximum dose in most cases is, however, $0.75 \mathrm{gm}$.

Indications for ceasing Treatment.-In my opinion treatment should not be stopped until the point is reached, when the secondary symptoms would have appeared if there had been no treatment. This represents roughly a date three months after that of infection. I consider it best, however, to stop, if possible, after $7 \frac{1}{2} \mathrm{gm}$., or 50 doses, have been given.

I object to the use of bismuth and mercury ; treatment may have to be suspended altogether if they produce, as they often do, mercurial stomatitis, nephritis, etc., and interfere with the effect of the salvarsan.

I advise an examination of the blood at the time of each injection, as I place great reliance on frequent bloodtests as a guide to the course of the disease.

If necessary the foregoing scheme is repeated after six months. Treatment is continued until a monthly blood test has shown a negative reaction. Patients are kept under observation for five years after the cessation of treatment before they are pronounced cured, during which time their blood serum is tested at intervals of one to three months.

Provocative injections are not given in this clinic.

The cerebro-spinal fluid is examined at the end of three years, but this test is sometimes considered unnecessary.

Primary syphilis, where the serum reaction is positive, and secondary syphilis, are treated in the same way except that a course of bismuth treatment is administered after the salvarsan.

Tertiary syphilitic cases receive iodine or bismuth, or both, in addition to salvarsan.

Latent syphilis is treated in the same way until the Wassermann reaction becomes negative.

Pregnant women suffering from syphilis are given a course of salvarsan at the third and at the eighth month.

In syphilis of the central nervous system, tabes and general paralysis everything is tried that is likely to have any effect. Bismuth treatment is given first place.

In late cases of syphilis, when the serum reaction is resistant, injections are continued until it becomes negative, no matter how long this may take. 


\section{BRITISH JOURNAL OF VENEREAL DISEASES \\ B. The Treatment of Gonorrhea}

General Treatment.-I treat gonorrhœa with antiseptic silver preparations and the proprietary vaccine "gonargin " regulating the dosage by the local reaction.

Local Treatment.-Injections of silver nitrate are administered with a syringe by both surgeon and patient. The surgeon gives one each day and the patient gives himself five every day between consultations. The dilution ordered varies between $I$ in 5,000 and I in $I, 000$. Daily irrigations are given by the surgeon, using the same salt in the same dilution ; the preparation " albargin" is that chiefly employed. The solution is never allowed to enter the bladder.

Chronic cases receive the same treatment, and prostatic infection, whether abscess is present or not, is dealt with by massage.

Epididymitis is treated with the wet pack and the ice bag ; arthritis, teno-synovitis and bursitis with hot applications, ichthyol and vaccines.

No patient is considered cured until he has given negative reactions for a month to tests for infection of the seminal vesicles and urethral glands.

\section{GONORRHCA IN WOMEN}

The treatment for women is identical with that given to men. Infection of the urethra, Bartholinitis and glandular gonorrhœea are found to be readily amenable. In gonorrhœa of the cervix, uterus and rectum, however, a year's probation is considered necessary before a patient can be finally discharged. 